\title{
Host galaxies of bright high redshift quasars: luminosities and colours ${ }^{\star}$
}

\author{
M. Schramm ${ }^{1}$, L.Wisotzki ${ }^{1}$, and K. Jahnke ${ }^{2}$ \\ 1 Astrophysikalisches Institut Potsdam, An der Sternwarte 16, 14482 Potsdam, Germany \\ e-mail: mschramm@aip.de \\ 2 Max-Planck-Institute for Astronomy, Königstuhl 17, 69117 Heidelberg, Germany
}

Received 16 February 2007 / Accepted 6 September 2007

\begin{abstract}
We present the results of a near-infrared imaging study of high redshift $(z \sim 3)$ quasars using the ESO-VLT. Our targets were selected to have luminosities among the highest known (absolute magnitude $M_{B} \lesssim-28$ ). We searched for resolved structures underlying the bright point-source nuclei by comparing the QSO images with stars located in the same fields. Two QSOs (HE 2348-1444 at $z=2.904$ and HE 2355-5457 at $z=2.933$ ) are clearly resolved in $K_{\mathrm{s}}$, and with somewhat lower significance also in $H$; one object is resolved only in $K_{\mathrm{s}}$. At these redshifts, $H$ and $K_{\mathrm{s}}$ correspond almost exactly to rest-frame $B$ and $V$, respectively, with virtually no $K$-correction. We also report the non-detection of some additional QSOs. The detected host galaxies are extremely luminous with $M_{V} \sim-25$. Their rest-frame $B-V$ colours, however, are close to zero in the Vega system, indicating substantial contributions from young stars and a stellar mass-to-light ratio below 1 (in solar units). Tentatively converting $M_{V}$ and $B-V$ into rough estimates of stellar mass, we obtain values of $M_{\star}$ in the range of several $10^{11} M_{\odot}$, placing them within the high-mass range of recent high-redshift galaxy surveys. We present optical spectra and use C IV line width measurements to predict virial black hole masses, obtaining typical values of $M_{\mathrm{BH}} \sim 5 \times 10^{9} M_{\odot}$. With respect to the known correlation between host galaxy luminosity $L_{V, \text { host }}$ and $M_{\mathrm{BH}}$, our measurements reach higher luminosities and redshifts than previous studies, but are consistent with them. Comparing our objects with the local $(z \simeq 0$ ) $M_{\mathrm{BH}}-M_{\text {bulge }}$ relation and taking also the low stellar mass-to-light ratios into account, we find tentative evidence for an excess in the $M_{\mathrm{BH}} / M_{\text {bulge }}$ mass ratio at $z \sim 3$.
\end{abstract}

Key words. galaxies: active - galaxies: high redshift - quasars: general

\section{Introduction}

Our understanding of quasar host galaxies has made rapid and significant progress over the past decade. It is now clear that luminous quasars $\left(M_{B} \lesssim-24\right)$ at low redshifts are mostly hosted by massive elliptical galaxies (McLure et al. 1999; Dunlop et al. 2003), irrespective of radio power. Nuclear and host bulge luminosities are correlated (McLeod \& Rieke 1995), which is naturally explained by the tight relation between the masses of supermassive black holes and bulges (e.g., Häring \& Rix 2004, and references therein), in particular if quasar black holes typically accrete and radiate at a fixed fraction of the Eddington rate (McLeod et al. 1999).

High redshift quasar hosts have also received increased attention lately, as they provide one avenue to study the assembly and evolution of massive galaxies, in particular in relation to the growth of their central black holes. Yet, quantitative knowledge of QSO host properties at redshifts well beyond $z \gtrsim 1$ is still very limited, mainly due to the fact that optical imaging only gives access to the rest-frame ultraviolet spectral range where the QSO nuclei outshine any host galaxy. The improvements in nearinfrared instrumentation have also opened this window, first with the Hubble Space Telescope (Kukula et al. 2001; Ridgway et al. 2001), followed by some ground-based experiments (Falomo et al. 2004, 2005; Kuhlbrodt et al. 2005). Very useful insights

* Based on observations with the European Southern Observatory Very Large Telescope (ESO-VLT), observing run ID P69.B-0118(A). have been obtained from HST imaging of gravitationally lensed QSOs (Peng et al. 2006b).

These studies were all limited to single band observations, typically sampling the rest-frame optical wavelength range. Largely focused on solving the challenging problem of resolving the QSO images and deblending the nuclear and underlying extended galaxy components, their main result is that QSOs are hosted by fairly luminous galaxies already at high $z$. More quantitatively, Kukula et al. (2001) found essentially no evolution between $z \simeq 2$ and $z \simeq 0$ in typical host luminosities, for radio-quiet QSOs at given absolute nuclear magnitude of $M_{B} \sim-24$. This trend has been confirmed by more recent studies based on larger samples (e.g. Peng et al. 2006a,b).

Unfortunately, additional information such as morphological properties or colours are even harder to come by than single band luminosities. Colours or more generally speaking, spectral data are already very scarce at much lower redshifts, and significant improvements have only been obtained in the last few years, at $z \lesssim 0.2$ (Jahnke et al. 2004a) and at $z \simeq 0.7$ (Sánchez et al. 2004). These studies have demonstrated that there is strong evidence for the presence of of blue, young stars in low-redshift QSO host galaxies, including those that appear to be quiescent ellipticals.

One would expect recent or ongoing star formation to be even more prevalent in galaxies at high redshifts. Indeed we found substantial UV emission from the hosts of moderate luminosity QSOs at $z \simeq 2.2$ using HST/ACS imaging (Jahnke et al. $2004 \mathrm{~b})$. But to our knowledge, no measurements of rest-frame 


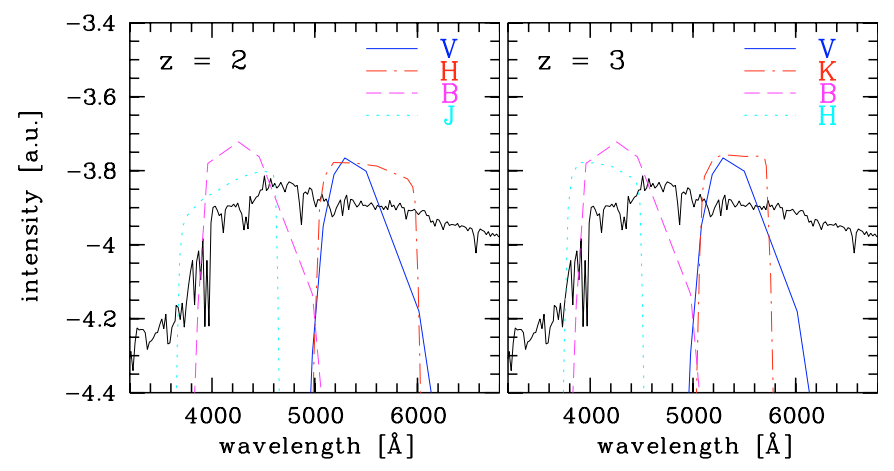

Fig. 1. Position of the corresponding filter profiles at $z=2$ and $z=3$. In both panels, the spectral template represents a single stellar population model of a 1 Gyr old galaxy.

optical colours have yet been published for high-redshift QSO host galaxies.

In the present paper we report on the results of a pilot study aimed at measuring host galaxy luminosities in two different near-infrared bands using the ESO-VLT. Our targets are radioquiet, very high luminosity QSOs at $z \sim 2$ and $z \sim 3$. We briefly describe our strategy to decompose the QSO images into their nuclear and host contributions, and we discuss the results in the context of recently obtained scaling relations. Throughout this paper we assume a flat cosmology with $H_{0}=70 \mathrm{~km} \mathrm{~s}^{-1} \mathrm{Mpc}^{-1}$, $\Omega_{\mathrm{M}}=0.3$ and $\Omega_{\Lambda}=0.7$. All magnitudes are expressed in the Vega system.

\section{Observations and data reduction}

\subsection{Target selection}

Our aim was to observe quasars with the highest possible luminosities, which we expect to reside in the most massive bulge-dominated galaxies. We used the Hamburg/ESO Survey for bright QSOs (HES; Wisotzki et al. 2000, Wisotzki et al., in prep.) as the input catalogue. Our QSOs were selected to have redshifts $2.6<z<3.0$ so that the rest-frame $B$ and $V$ bands were approximately reproduced by the standard near-infrared filters $H$ and $K_{\mathrm{s}}$, respectively. We also added a few QSOs with $1.8<z<2$, where $J$ and $H$ correspond to rest-frame $B$ and $V$, respectively. Figure 1 illustrates the good match between restframe and observed-frame filter bands. We verified by visual inspection in the Digitized Sky Survey that there was at least one star of comparable brightness within $\sim 1$ arcmin of each QSO, which later would be usable for calibrating the point-spread function. Table 1 lists the small sample of objects for which we obtained useful data.

\subsection{Observations}

The sample was observed with the ESO-VLT in service mode (thus spreading over several nights) between May and July 2002. The instrument used was the Infrared Spectrograph and Array Camera (ISAAC), mounted on the Nasmyth B focus of UT1 (Antu) of the VLT. Because of technical problems with the short waveband ("Hawaii") arm, only the long waveband arm was available. This was equipped with a SBRC Aladdin $1024 \times$ 1024 pixel InSb array providing a pixel scale of $0 . ' 148$ pixel $^{-1}$ for a total field of view of $151^{\prime \prime} \times 151^{\prime \prime}$. The data were obtained in observing blocks consisting of sequences of 12 exposures in $J$, 15 in $H$ and 30 in the $K_{\mathrm{s}}$ band. In order to account for the rapidly varying NIR night sky, the telescope was moved between successive exposures following a multi-point dither pattern along a sequence of predefined quasi-random grid points. Exposure times per dither pointing were 150,120 and $60 \mathrm{~s}$ in $J, H$ and $K_{\mathrm{s}}$, respectively, each split into several short integrations. The final net integration times per target and filter band are listed in Table 1, along with the effective seeing measured in the coadded images. All observations were obtained under photometric conditions.

\subsection{Data reduction}

The images were reduced using ESO-MIDAS and the eclipse software package also provided by ESO, in particular the eclipse-based ISAAC reduction pipeline recipe $1 w j$ itter. The basic steps involved (i) flat fielding using twilight flats, (ii) estimate of a sky frame by median filtering of the individual frames in the stack, after rescaling to account for temporal sky level variations, (iii) sky subtraction, (iv) determination of the angular offsets between frames, using an auto-correlation technique, (v) final image registration and coaddition for each stack of images.

As for all observations in the NIR, our data are totally dominated by the sky background. The background removal by the reduction pipeline was not quite good enough for a detailed analysis of very low surface brightness features. We therefore determined a local background correction for each object separately by creating a curve of growth. This generally worked satisfactorily, although the data sometimes suffered from spatial background features varying with time, which we could not correct for. The main effect of such variations was an increase of the statistical uncertainty of the local background correction. We quantified this uncertainty by extracting several small control images of blank sky in arbitrary positions, which were then treated in the same way.

\subsection{Photometry and absolute magnitudes}

Flux calibration was based on observations of standard stars from Persson et al. (1998). Atmospheric extinction coefficients were taken from the ESO web pages. The derived zero points from various stars turned out to be highly consistent, with a median internal error of 0.003 mag.

Conversion of apparent into absolute magnitudes requires knowledge of a $K$-correction (including the band pass stretching). Given the design of our experiment, the spectral corrections are very small (cf. Fig. 1). We followed in principle the approach described by Jahnke et al. (2004a). For the K-correction of the nucleus we used an average quasar SED, for the host galaxies we calculated K-corrections from single stellar population models with stellar ages ranging between $0.1-1$ Gyr. The resulting absolute magnitudes, however, are almost independent of the assumed spectral energy distributions.

\section{Analysis}

\subsection{Point spread function}

The detection of faint extended fuzz underlying a bright point source is notoriously difficult and requires an accurate knowledge of the effective point spread function (PSF) of the optical system. All quasars in the sample were selected to have at least one suitable PSF star in their vicinity. Unfortunately, a detailed analysis of stellar profiles within the full ISAAC field of view revealed significant spatial variations of the PSF, reminiscent of optical aberrations. Even worse, the pattern clearly also varied in 
Table 1. Target list and log of observations.

\begin{tabular}{cccccccccccc}
\hline \hline source & \multirow{2}{*}{ RA } & Dec & \multirow{2}{*}{ R } & \multirow{4}{*}{ Exp. time [min] } & \multicolumn{3}{c}{ seeing [arcsec] } \\
& & & & & $J$ & $H$ & $K_{\mathrm{s}}$ & $J$ & $H$ & $K_{\mathrm{s}}$ \\
\hline HE 2329-4115 & 233150.5 & -405853 & 1.850 & 18.7 & 60 & 30 & - & 0.75 & 0.70 & - \\
HE 2213-3722 & 221630.8 & -370732 & 1.920 & 17.6 & 30 & 60 & - & 0.71 & 0.73 & - \\
HE 2149-0436 & 215150.1 & -042219 & 2.643 & 17.3 & - & 60 & 60 & - & 0.58 & 0.71 \\
HE 2355-5457 & 235833.5 & -544042 & 2.904 & 17.8 & - & 90 & 90 & - & 0.73 & 0.70 \\
HE 2348-1444 & 235129.8 & -142757 & 2.933 & 17.3 & - & 60 & 60 & - & 0.65 & 0.65 \\
\hline
\end{tabular}

time, possibly due to focus variations, and no two images shared the same pattern. While in principle it is possible to model such distortions using several stars in the field of view (e.g., Kuhlbrodt et al. 2004), the number of stars at these high Galactic latitudes was mostly too small. Some of our images thus became useless, with the PSF stars displaying a broader radial profile than the QSOs. Such data had to be discarded entirely, and they are not discussed further in this paper.

In the end, we used only exposures where the PSF appeared to be reasonably stable within the central regions. In our best cases we had two stars close to the quasar that were bright enough to be useful as PSF calibrators; here we used either an average PSF for each given image, or each star individually when explicitly quantifying the effects of PSF uncertainty. In particular we adopted the difference image of the two stars as a rough guess of the additional "noise" contributed by the PSF uncertainty.

\subsection{PSF subtraction}

PSF subtraction is a well-established technique for the analysis of QSO host galaxy images. It has the advantage that a minimum of assumptions about the nature of the host enters the analysis. Here we employed it with the main purpose of performing a conservative test for the "null hypothesis", namely that no resolved host galaxy is detectable underneath the QSO point source.

Once a PSF was established, it had to be shifted to the location of the QSO, scaled, and subtracted. To minimize shifting residuals we used a subpixel grid for the centroid position and found the best location from $\chi^{2}$ minimisation. As a scaling criterion we demanded that the summed residual flux in a small aperture of 2 pixels radius around the centroid vanished after PSF subtraction. Unresolved sources can thus be robustly identified, as any residual flux after PSF subtraction should be consistent with PSF mismatch errors. On the other hand, it is obviously a very conservative criterion for resolved sources implying a significant oversubtraction if a host galaxy were present. However, a significantly detected extended source should still yield systematically positive residuals at larger radii. Only those detections were taken as significant that show positive residuals for most of the bright stars that are located in the vicinity of the QSO and contain at least 5\% of the QSO flux after oversubtraction correction. In such cases it is even possible to approximately account for the oversubtraction by applying a correction factor derived from simulations (see Jahnke et al. 2004b, for a more extensive discussion of this procedure). Briefly, we performed a series of such simulations where artificial host galaxies were added to one of the PSF stars. After adding appropriate noise we took a second star in each field as PSF calibrator which was shifted, scaled, and subtracted. This provided us with a rough estimate of the systematic PSF oversubtraction. The model host galaxies in this exercise were assumed to be smooth and circularly symmetric, with a surface brightness distribution described by a de Vaucouleurs profile of effective radius of 3, 5 and $10 \mathrm{kpc}$, respectively. This is slightly more compact than present-day highmass elliptical galaxies, but plausible given the high redshifts involved and given earlier results (see also Kuhlbrodt et al. 2005). While typical values of the residual flux are of the order of a few percent we determined oversubtraction correction factors of $0.5-1 \mathrm{mag}$ for the $K_{\mathrm{s}}$ band and somewhat higher values of 1-1.5 mag in the case of the $H$ band. We obtained similar correction factors if an exponential disk is assumed instead of a de Vaucouleurs profile as the galaxy model. We find the apparent host galaxy magnitudes to be fainter by $0.2-0.5 \mathrm{mag}$ and stronger residuals at the same time. After the oversubtraction correction was applied, all nuclear and host galaxy magnitudes agree within 0.2 mag with the values determined by our two-dimensional modelling technique. A similar procedure as described above was applied to the non-detections to determine upper limits of the host luminosity. Here we created series of quasar images by adding a synthetic galaxy component of 3, 5 and $10 \mathrm{kpc}$, respectively, to the given PSF. We varied the nuclear/host flux ratio until we clearly resolved the galaxy in the radial surface brightness profile. We adopted upper limits when the quasar surface brightness deviated from the PSF profile by $3 \sigma$ based on shot noise error bars determined in a ring excluding the central pixels.

\subsection{Two-dimensional modelling}

A more advanced deblending treatment is based on the explicit assumption that the surface brightness profile of an observed host galaxy should roughly follow a simple analytical prescription. It is then possible to fit a model of the two-dimensional light distribution to the data, consisting of a superposition of a scaled PSF plus an analytical galaxy model convolved with the PSF. We used our own code PAMDAI (Kuhlbrodt et al. 2004) which in the past was successfully applied to low-redshift QSO hosts (e.g., Jahnke et al. 2004a). The code determines the best-fit parameters by $\chi^{2}$ minimisation employing a modified downhill simplex algorithm.

We soon realised that the signal of any possible host galaxy was too weak in our data to permit simultaneous constraining of the shape and the flux level of the galaxy model. We therefore made the following additional assumptions: The host galaxy was assumed to follow a de Vaucouleurs law with zero ellipticity and with a preset effective radius $r_{\text {eff }}$. When assuming an exponential law instead, we find the host magnitudes to be fainter by 0.3-0.4 mag. Thus, only the fluxes of the nucleus and galaxy had to be determined by the fit. With these provisions the fits converged well for those objects where the PSF subtraction indicated a resolved source. It turned out that the $\chi^{2}$ values resulting from assuming different fixed values of $r_{\text {eff }}$ were very similar as long as realistic $r_{\text {eff }}$ values were chosen. It is thus not possible to 


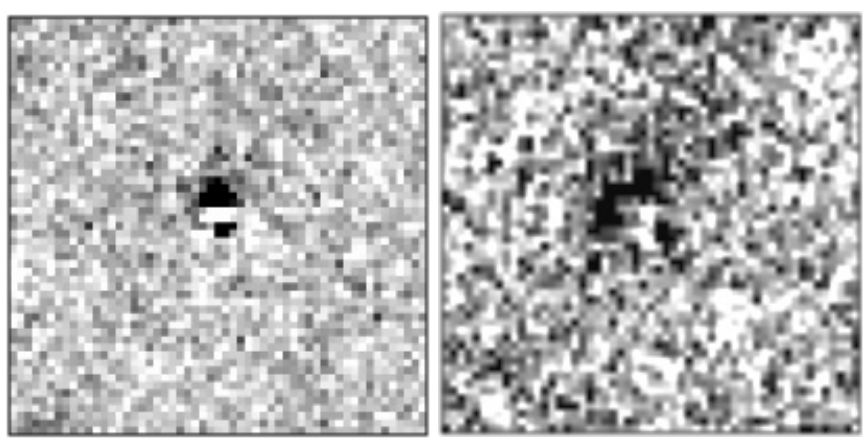

Fig. 2. Residuals after subtracting one PSF star from another star for HE 2355-5457 (left) and HE 2348-1444 (right). We used the two stars marked as S1 and S2 in Fig. 3. Cuts are chosen the same as for Fig. 3.

significantly constrain the scale length of the host galaxy from our data, which is perhaps not too surprising as our angular resolution of 0.7 corresponds to $\sim 5 \mathrm{kpc}$ at $z=3$. However, the estimated host galaxy fluxes do not depend sensitively on the correct scale length. This fact has been known for long (Abraham et al. 1992), and we confirm it to be valid for our present data, also through extensive simulations.

We computed a two-dimensional model only in those cases where the conservative PSF subtraction revealed positive residuals. We then adopted the best-fit PSF scaling factor to obtain a best-guess PSF-subtracted image of the host galaxy.

\section{Results}

We resolved the host galaxies of three QSOs from the higher redshift subsample, two of them in both $H$ and $K_{\mathrm{s}}$ bands, and one only in $K_{\mathrm{s}}$. We failed to detect the hosts of two QSOs from the lower redshift subsample. We now briefly discuss the sources individually. A summary is given in Table 2, images of the detected QSOs are shown in Fig. 3.

HE 2355-5457: This field has two nearby stars of comparable brightness to the QSO located within less than $45^{\prime \prime}$ distance from the QSO. There is no evidence for significant PSF variations over the central regions of the field. Subtracting a composite scaled PSF from the QSO left a strong excess of positive pixel values outside the central zone, in both $H$ and $K_{\mathrm{s}}$ bands. We tested the reality of this effect by subtracting one PSF star separately from either the QSO or the other star. The difference QSO-PSF consistently showed a $10 \times$ higher residual flux than the difference of PSF1-PSF2. Visual inspection of the PSF-subtracted QSO image showed that this excess in the quasar profile was not caused by a close companion, or by a strongly asymmetric feature, but that it was due to more or less azimuthally symmetric low surface brightness emission centered on the QSO. We conclude that our conservative null hypothesis of the QSO having no detectable host galaxy has to be rejected, with high significance for the $K_{\mathrm{s}}$ band, but also (with somewhat lower significance) for the $H$ band. However, with the galaxy contributing only $~ 5 \%$ of the total uncorrected $K_{\mathrm{s}}$ band flux, the host is still much fainter than the central nucleus. In the $H$ band this fraction is even slightly less than $3 \%$. After applying a statistical correction for oversubtraction (see Sect. 3.2) these values increase to $\sim 10 \%$ and $\sim 8 \%$ for the $K_{\mathrm{s}}$ and $H$ band, respectively. We then computed two-dimensional model fits (with fixed scale lengths as discussed above), and obtained integrated $K_{\mathrm{s}}$ and $H$ band fluxes for the host galaxy that are in good agreement with the corrected estimates based on PSF-subtraction. This makes HE 2355-5457 the QSO with the highest significance detection in the whole sample. The results are documented in the upper panels of Fig. 3.

HE 2348-1444: The situation with this QSO is similar to the previous object: We have two bright stars close to the quasar which show very similar surface brightness profiles. We again find positive PSF-subtraction residuals in both bands which were confirmed by the same test procedure as described for HE 2355-5457. However, the fraction of an extended flux component is even lower, only $1-2 \%$, or $6 \%$ and $5 \%$ in $K_{\mathrm{s}}$ and $H$, respectively, after correction for oversubtraction. Notice that HE 2348-1444 is the brightest quasar in our sample. In this object the residual flux is strongly asymmetric (see Fig. 3). This feature becomes particularly evident after subtracting the azimuthally symmetric two-dimensional model fit. At the same time, the lacking symmetry makes the extended nature of the object inconspicuous in the radial profiles (it is not visible in the $H$ band profile). Nevertheless, we believe that since the feature appears as highly consistent in both bands, its reality can be safely assumed. We conclude that also in this QSO we have detected the host galaxy, and that there is evidence for a highly disturbed morphological structure, most likely due to gravitational interaction or merging.

HE 2149-0436: The field contains several (mostly faint) stars showing that PSF variations for the whole field of view are rather large. For both bands we find positive as well as negative residuals after subtraction of individual stars extracted within $30^{\prime \prime}$ around the QSO. In the $K_{\mathrm{S}}$ band, the number of sufficiently bright field stars was acceptable to create an analytical model of the spatial PSF variations (similar to the procedure of Kuhlbrodt et al. 2004). Using the best-fit analytical PSF prescription at the QSO position, we detected an extended source after PSF subtraction. The amount of uncorrected flux is $6 \%$ and increases to $13 \%$ after oversubtraction correction. The image quality of the $H$ band data was unfortunately inferior to the $K_{\mathrm{s}}$ data, and we did not succeed in resolving the host galaxy. In the following we only quote a $3 \sigma$ upper limit of the host galaxy luminosity, propagating into corresponding lower limits of the rest-frame $B-V$ colour.

HE 2329-4115 and HE 2213-3722: While the $J$ band data of these $z \sim 2$ QSOs turned out to be useless, we found both QSOs to be marginally resolved in the $H$ band, but below our formal significance criterion. We therefore only quote $3 \sigma$ upper limits in Table 2.

\section{Discussion}

\subsection{Host galaxy luminosities}

The fluxes of the detected host galaxies were determined by integrating over the PSF-subtracted images, where the PSF scaling factor was given by the best-fit analytic model. Since we did not allow the half-light radii to vary during the fit, the resulting fluxes depend somewhat on the assumed size of the galaxy. We found that our adopted range for $r_{1 / 2}$ from $3 \mathrm{kpc}$ to $10 \mathrm{kpc}$ corresponds to a systematic uncertainty of \pm 0.2 mag, regardless of the filter used. In those cases where only upper limits are quoted, the conservatively PSF-subtracted images were used. 
Table 2. Integrated apparent magnitudes and absolute magnitude estimation for the observed QSOs. The results are quoted for a fixed effective radius of $5 \mathrm{kpc}$.

\begin{tabular}{lrccccccc}
\hline \hline Object & \multicolumn{1}{c}{$H^{\text {host }}$} & $H^{\text {nuc }}$ & $K_{\mathrm{s}}^{\text {host }}$ & $K_{\mathrm{s}}^{\text {nuc }}$ & $M_{B}^{\text {nuc }}$ & $M_{B}^{\text {host }}$ & $M_{V}^{\text {nuc }}$ & $M_{V}^{\text {host }}$ \\
\hline HE 2149-0436 & $>18.0$ & 16.3 & 18.0 & 15.9 & -27.4 & $>-25.6$ & -27.4 & -25.4 \\
HE 2355-5457 & 18.0 & 15.8 & 17.8 & 15.5 & -28.2 & -25.8 & -28.2 & -26.0 \\
HE 2348-1444 & 17.8 & 14.8 & 17.4 & 14.2 & -29.3 & -26.4 & -29.5 & -26.3 \\
HE 2213-3722 & $>18.5$ & 16.6 & & & & & -26.7 & $>-24.8$ \\
HE 2329-4115 & $>17.8$ & 16.9 & & & & & -26.3 & $>-25.4$ \\
\hline
\end{tabular}

After applying distance modulus and $K$ corrections as discussed above, we obtained absolute magnitudes in the restframe $V$ and $B$ bands. We reiterate that our measurements essentially sample the objects at these rest-frame wavelengths, and that $K$ corrections are almost negligible. The resulting absolute magnitudes are listed in Table 2 . Notice that the quasars in our sample are so powerful that despite the high nuclear-host ratio of $\gtrsim 10$, the hosts are still extremely luminous galaxies.

Figure 4 compares our measurements to other recent studies of high-redshift QSO host galaxies. We restrict this comparison to near-infrared studies where $M_{V}$ of the host galaxy can be estimated without major spectral extrapolation. While evidently there are very few detections for high-luminosity QSOs, our data are consistent with the published observations. Figure 4 suggests that QSO nuclear and host galaxy luminosities also are correlated at high redshifts, very similar to the finding by Floyd et al. (2004) at low $z$.

QSOs radiating at a fixed fraction of the Eddington limit are distributed along a diagonal in this diagram (McLeod et al. 1999), assuming that black hole masses are proportional to host bulge luminosities (Magorrian et al. 1998). Under these premises, our objects appear to have similar Eddington ratios as less luminous QSOs at redshifts $z \gtrsim 2$ (and also similar to low- $z$ QSOs). This very qualitative reasoning can be quantified if mass estimates are available for both black holes and stellar bulges. In the following we attempt to estimate both these quantities for our $z \sim 3$ QSOs.

\subsection{Black hole masses}

Several recent investigations have demonstrated that one can estimate the masses of black holes in active galactic nuclei from single-epoch spectra of broad emission lines (Vestergaard 2002; Vestergaard \& Peterson 2006; McLure \& Jarvis 2002). This approach assumes the broad-line region (BLR) to be in approximate virial equilibrium (Peterson \& Wandel 2000) and adopts an empirical luminosity-BLR size scaling relationship (Kaspi et al. 2000). The resulting object-to-object scatter is substantial, but there is no systematic bias (Collin et al. 2006). We adopt the prescription used by Vestergaard \& Peterson (2006) to compute black hole masses directly from observables,

$M_{\mathrm{bh}}=5.4 \times 10^{6}\left\{\left[\frac{\sigma_{1}(\mathrm{C} \mathrm{IV})}{1000 \mathrm{~km} \mathrm{~s}^{-1}}\right]^{2}\left[\frac{\lambda L_{\lambda}(1350 \AA)}{10^{44} \mathrm{erg} / \mathrm{s}}\right]^{0.53}\right\}$

where $L_{\lambda}(1350 \AA)$ is the monochromatic luminosity at $1350 \AA$, and $\sigma_{l}(\mathrm{CIV})$ is the dispersion (second central moment) of the C IV $\lambda 1550$ emission line.

From the follow-up spectroscopy within the Hamburg/ESO Survey we have slit spectra available of all three QSOs where we resolved the host galaxies. These spectra are presented in Fig. 5.
We measured the C IV line dispersion in the following way: We first subtracted a local continuum, estimated as a straight line fitted to the line-free windows at $1440 \AA<\lambda<1480 \AA$ and $1660 \AA<\lambda<1700 \AA$; a slight extrapolation of this also provided the continuum flux at $1350 \AA$. We then determined the second central moment $\sigma_{1}$ for each line. Two of our objects (HE 2348-1444 and HE 2355-5457) show evidence of associated absorption in the blue wing of C IV for which we had to correct. In HE 2355-5457 it was straightforward to interpolate over the absorption line, whereas in HE 2348-1444 some guesswork was required, given the low spectral resolution. Fortunately, $\sigma_{1}$ is not very sensitive to such manipulations (unlike the FWHM), and the difference between the best-guess corrected and completely uncorrected $\sigma_{1}$ was only $\sim 10 \%$, which would translate into a $20 \%$ change in $M_{\mathrm{bh}}$. As some degree of correction was undoubtedly needed, we argue that the additional error introduced this way is smaller. The results are presented in Table 3.

As expected given the high luminosities, the black holes are very massive with $M_{\mathrm{bh}}$ up to a few times $10^{9} M_{\odot}$. Yet, these masses are well within the upper envelopes of the distribution obtained by Vestergaard (2004) for high-luminosity highredshift QSOs. In Fig. 6 we investigate where our objects fall within the $M_{\mathrm{bh}}-L_{V}$ relationship, compared to other high redshift QSOs with detected host galaxies and available black hole mass estimates. (In this context we use the term $L_{V}$ to avoid confusion between masses and absolute magnitudes; nevertheless we always express luminosities in terms of absolute magnitudes $M_{V}$.) Our three objects are consistent with an approximately linear $M_{\mathrm{bh}}-\log L_{V}$ relation.

A revised prescription of the $M_{\mathrm{bh}}-L_{V}$ relation for lowredshift bulges and ellipticals was derived by Dunlop et al. (2003) as $M_{\mathrm{bh}}=1.3 \times 10^{-6} L_{\text {bulge }}^{1.31}$, in solar units. We overplot this relation as a dashed line in Fig. 6 . There is a surprisingly good agreement between the low- $z$ relation and the high- $z$ data, which at face value might indicate no evolution at all. Of course, this cannot be true.

Applying the above formula explicitly assumes a welldefined mass-to-light ratio for QSO host galaxies, essentially that of old elliptical galaxies at $z \sim 0$ (Jorgensen et al. 1996). Overplotting this relation on a diagram showing data obtained at $z>1.5$ up to $z \sim 3$, without accounting at least for passive evolution, is therefore misleading. Only spectral information can reveal the amount of galaxy evolution required to match the high-redshift results to the local universe. Unfortunately, for most of the data shown in Fig. 6 there are only single-band measurements available. But since a substantial amount of evolution is unavoidable, Peng et al. (2006a) argued that even after conservatively correcting for only passive evolution with an assumed formation redshift of $z=5$, there must be a significant offset between the local and high-redshift relations. They concluded that at redshifts $z \sim 2$, the ratio of black hole to stellar bulge masses 


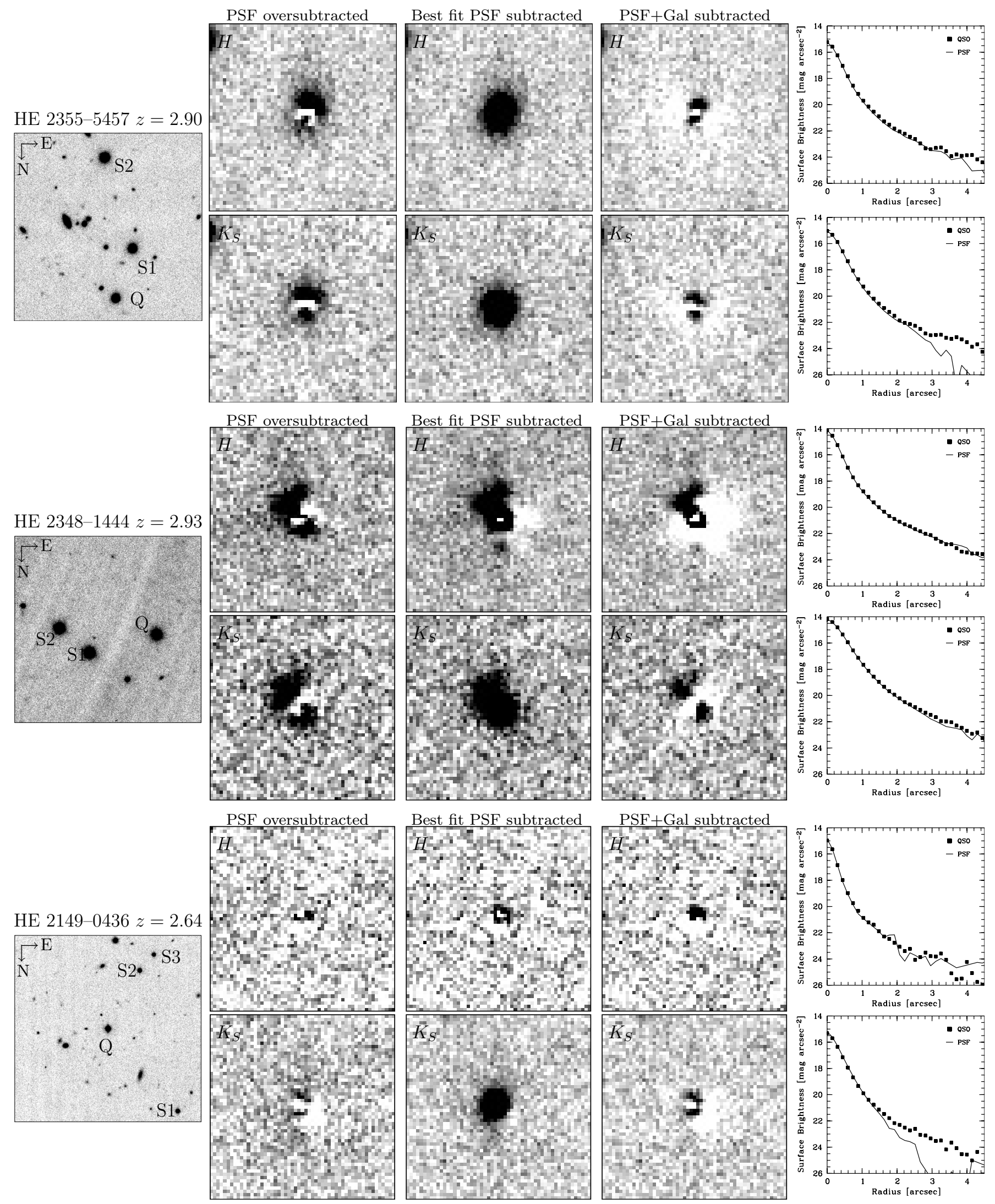

Fig. 3. Host galaxy detection of HE 2355-5457 (upper panel), HE 2348-1444 (middle panel) and HE 2149-0436 (bottom panel). The left-hand images have a scale of $1^{\prime} \times 1^{\prime}$ and show the QSO with PSF calibrator stars; for each QSO, $H$ band in the top row, $K_{\mathrm{s}}$ band below. All other images are $8^{\prime \prime} \times 8^{\prime \prime}$ enlargements centered on the QSO. The three middle panels show the residual host galaxy light after PSF subtraction; middle left: based on conservative PSF subtraction; center: based on the best-fit PSF scaling; middle right: residuals left after subtracting both the best-fit PSF and an azimuthally symmetric model host galaxy. In the right hand panels we show the radial surface brightness profiles of the QSO and corresponding best-fit PSF. 


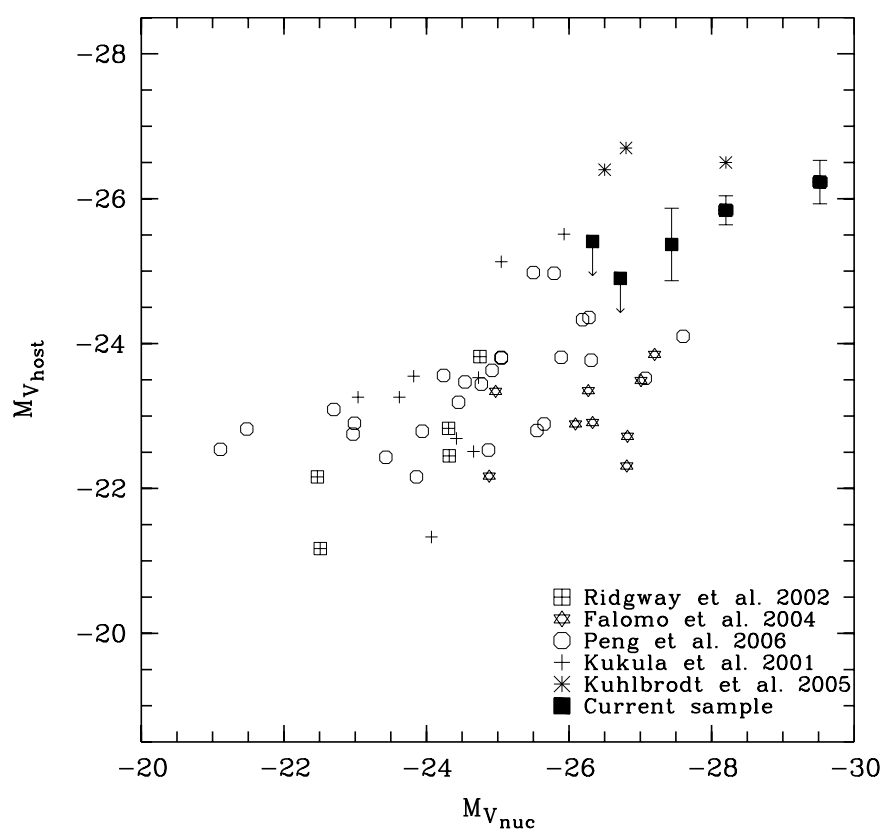

Fig. 4. Relation between nuclear and host galaxy luminosities for highredshift $(z>1.4)$ QSOs. Our data are represented by the filled squares. For comparison we plot several results from other recent studies (Ridgway et al. 2001; Kukula et al. 2001; Falomo et al. 2004; Kuhlbrodt et al. 2005; Peng et al. 2006a), all numbers converted to our set of cosmological parameters. Objects radiating at a constant fraction of their Eddington luminosity would lie on a diagonal line in this diagram.

must have been higher than "today" by at least a factor of 4 . We explore in the next subsection how our colour measurements fit into this picture.

Using our black hole masses we can also predict Eddington luminosities and Eddington ratios $\epsilon$ (cf. Table 3). We find that even these high-luminosity QSOs do not exceed Eddingtonlimited accretion, in fact their inferred Eddington ratios are completely consistent with those obtained for less luminous AGN at all redshifts (e.g., Kollmeier et al. 2006).

\subsection{Host galaxy colours: evidence for young stellar populations}

For the two QSO hosts detected in both $H$ and $K_{\mathrm{s}}$ we could also derive rest-frame $B-V$ colours; for the third object we obtained a conservative limit on $B-V$. We find that the colours of our two objects depend very little on the assumed scale lengths or morphology. The results are compiled in Table 3. The host galaxies are remarkably blue, with a $B-V$ of just below 0 (in the Vega system) with an uncertainty of $0.3 \mathrm{mag}$, including our individual uncertainties in the host magnitudes of about 0.2 mag and in the K-correction of about $0.05 \mathrm{mag}$ ) - thus corresponding to the colours of A-type stars. In terms of luminosity-weighted stellar ages (the ages of single-burst stellar populations (SSP) with the same $B-V$ ), we obtain values of $\sim 300 \mathrm{Myr}$ for both objects.

Finding that QSO host galaxies at $z \sim 3$ have blue colours and young stellar populations is not surprising, but it nevertheless poses relevant constraints. Note that a literal interpretation of the SSP ages leads to a formation redshift of $z \simeq 3.3$, considerably lower than the $z=5$ formation (conservatively) assumed by Peng et al. (2006a).

Blue colours of QSO host galaxies have recently been established as ubiquitous at lower redshifts. In Jahnke et al. (2004a)
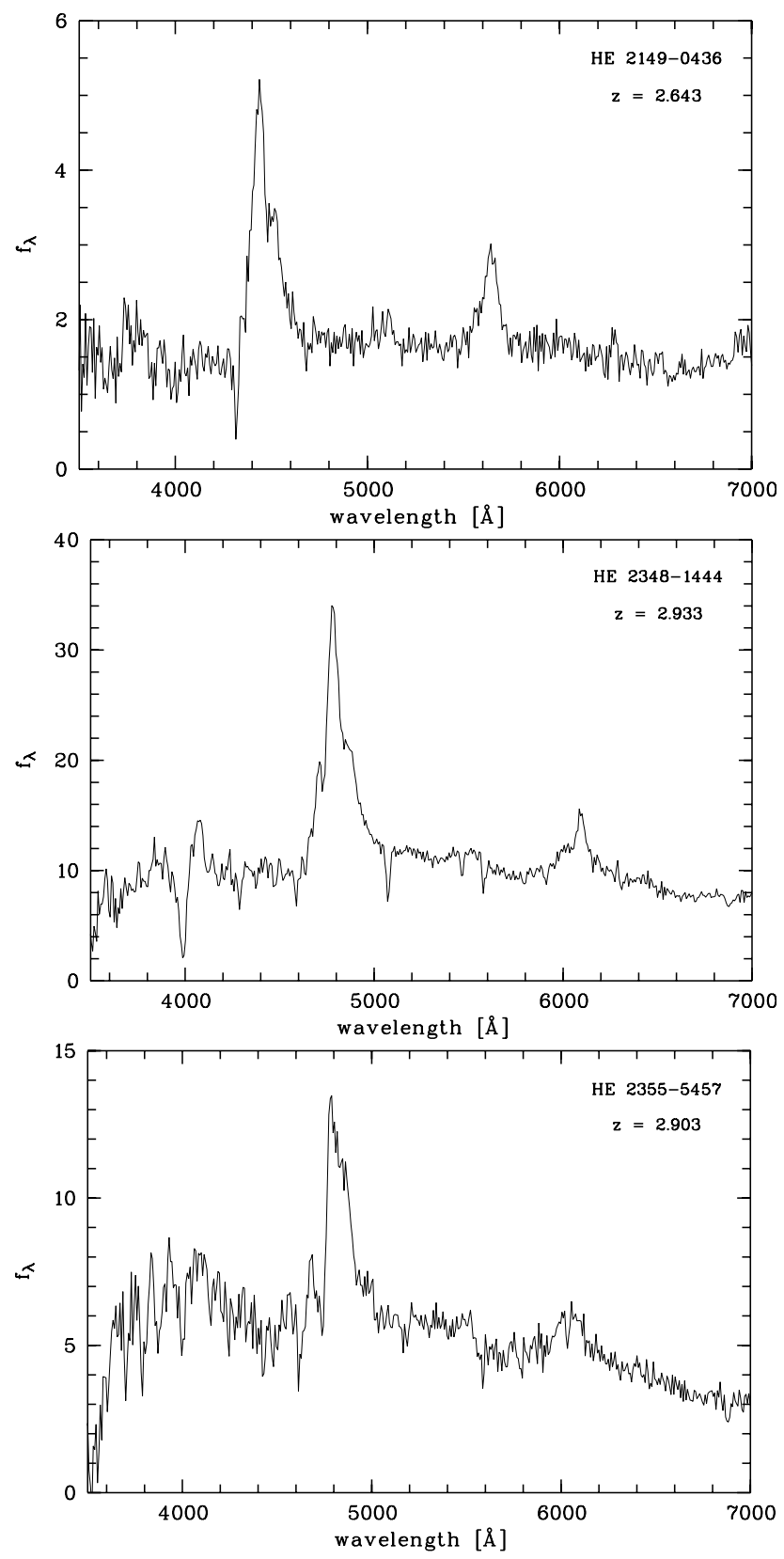

Fig. 5. Slit spectra of HE 2149-0436, HE 2355-5457 and HE 2348-1444. $f_{\lambda}$ is in units of $10^{-16} \mathrm{erg} \mathrm{cm}^{-2} \mathrm{~s}^{-1} \AA^{-1}$.

we demonstrated that $z<0.2$ quasar hosts with elliptical morphology have significant bluer colours than their inactive counterparts. Sánchez et al. (2004) presented a similar conclusion for a sample of QSO hosts at $z \simeq 0.7$ using HST imaging. In a different approach, Kauffmann et al. (2003) used SDSS data to show that Seyfert-2 galaxies are typically hosted by massive early-type galaxies with strong evidence for recent star formation. Comparing our new rest-frame $B-V$ values with those typically found at low redshifts, we find them to be even somewhat bluer but formally still consistent (given our error bars).

Corresponding data on high redshift QSO hosts are very scarce. From optical imaging of several $z>1.8$ QSOs with HST, Jahnke et al. (2004b) found evidence for substantial young stellar populations, with rest-frame UV luminosities comparable to Lyman Break Galaxies. We do not sample the rest-frame UV in our objects (and presumably would be overwhelmed by 
Table 3. Host galaxy and nuclear properties: Observed $H-K_{\mathrm{s}}$, rest-frame $B-V$, stellar mass $M_{\star}$, C IV emission line dispersion $\sigma_{1}$, C IV continuum fluxes at $1350 \AA$, estimated black hole mass $M_{\text {bh }}$, Eddington ratio $\epsilon$ and the black hole/bulge mass ratio $\mu$.

\begin{tabular}{lrrrrrrrr}
\hline \hline Object & $H-K_{\mathrm{s}}$ (obs.) & $B-V$ (rest) & $\begin{array}{r}M_{\star} \\
{\left[10^{11} M_{\odot}\right]}\end{array}$ & $\begin{array}{r}\sigma_{1} \\
\mathrm{~km} \mathrm{~s}^{-1}\end{array}$ & $\begin{array}{r}\log \left(\lambda L_{\lambda}\right) \\
\mathrm{erg} / \mathrm{s}\end{array}$ & $\begin{array}{r}M_{\mathrm{bh}} \\
{\left[10^{9} M_{\odot}\right]}\end{array}$ & $\epsilon$ \\
\hline HE 2149-0436 & $>0.03$ & $>-0.33$ & $>1.1$ & $2900 \pm 150$ & 46.7 & 1.2 & 0.3 & $<0.011$ \\
HE 2355-5457 & 0.26 & -0.16 & $2.6_{-1.8}^{+5.5}$ & $4100 \pm 400$ & 47.3 & 5.1 & 0.3 & $0.019_{-0.012}^{+0.064}$ \\
HE 2348-1444 & 0.39 & -0.07 & $4.9_{-3.3}^{+10.0}$ & $4300 \pm 300$ & 47.6 & 8.2 & 0.6 & $0.017_{-0.011}^{+0.055}$ \\
\hline
\end{tabular}

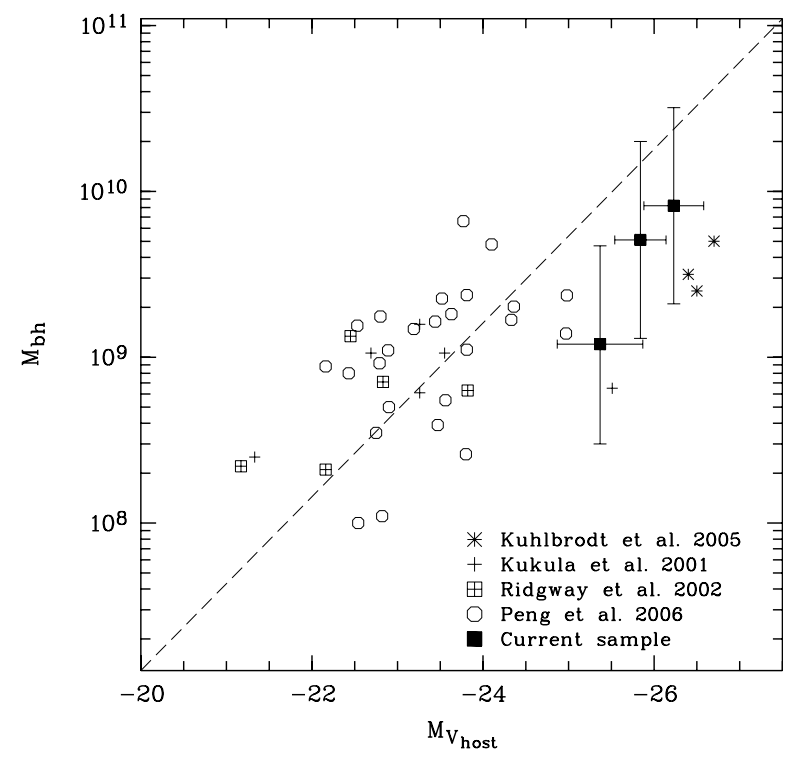

Fig. 6. Relation between black hole masses and host luminosities. Filled squares are our data; additional data points are taken from the literature. The dotted line shows the zero-redshift relation from Dunlop et al. (2003).

the nuclei anyway), so a direct comparison is not possible. We conclude that our finding of blue colours is qualitatively in very good agreement with most previous results.

\subsection{Stellar mass-to-light ratios and the $M_{b h}-M_{\text {bulge }}$ relation}

We can now return to improve our handling of the relation between black holes and their host galaxies. While there is no unique conversion of a single colour measurement into a stellar mass-to-light ratio, it is clear that the blue $B-V$ colours found for our objects imply rather low $M / L$. Bell et al. (2003) demonstrated that for a variety of star formation histories, stellar $M / L$ can be quite robustly predicted from $B-V$, and we used their formula

$\log _{10}\left(M / L_{V}\right)=-0.628+(1.305 \times(B-V))$.

where $M / L_{V}$ is given in solar units.

Using the $B-V$ colours of Table 3 , we obtained $M / L_{V}$ ratios in the range of $0.05-0.2$. Given our estimated uncertainties for the colours the associated uncertainties in $M / L_{V}$ are \pm 0.25 dex.

Combining our $M / L$ ratio estimates with the host galaxy luminsities above leads to stellar masses in the range of a few $10^{11} M_{\odot}$ for the two main objects. The formally computed values are listed in Table 3 . Thus, these galaxies are still very massive, but well within the range of the empirically samples stellar mass function of inactive galaxies as determined recently by Fontana et al. (2006).

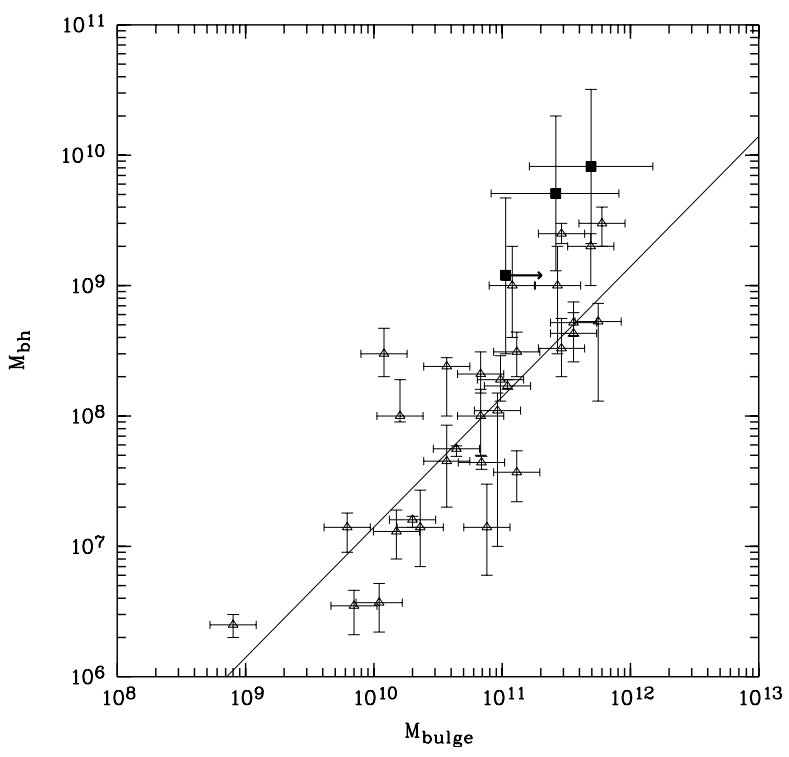

Fig. 7. $M_{\text {bh }}-M_{\text {bulge }}$ relation reproduced from Häring \& Rix (2004) for a sample of local galaxies. The solid line represents a constant mass ratio $M_{\mathrm{bh}} / M_{\text {bulge }}=0.0014$. The three filled symbols show our estimated stellar and virial black hole masses for the three $z \sim 3$ QSOs.

We then combined the black hole masses derived above with our estimated stellar masses. In doing so we assumed our luminous, massive host galaxies to be dominated by a bulgelike component. Our derived ratio $\mu$ between $M_{\text {bh }}$ and $M_{\text {bulge }}$ is given in Table 3; the two values and one upper limit are all $>0.01$. Formally, this is considerably larger than the local ratio of $\sim 0.0015$ obtained for inactive galaxies, although the discrepancy is only of the order of $1-2 \sigma$ given the substantial individual error bars. Note that any additional non-bulge component would increase the discrepancy.

We illustrate our results in Fig. 7 by overplotting our highredshift data points into the local $M_{\mathrm{bh}}-M_{\text {bulge }}$ relation obtained by Häring \& Rix (2004), together with a constant $\mu=0.0014$ relation (solid line). Our points are always above the local relation, although only marginally. Furthermore, the masses probed by our high-luminosity objects are at the extreme end of the range probed by the local data, and it is not yet clear that it can be extrapolated at constant $\mu$ into the high mass regime.

Measuring $M_{\mathrm{bh}} / M_{\text {bulge }}$ for different cosmological epochs, is presently beeing studied, and many groups have now endeavoured to estimate $\mu$ with a variety of different methods. Interestingly, most find evidence for $\mu$ to increase with lookback time. We already mentioned Peng et al. (2006a,b) who obtained $\mu_{z=2} / \mu_{z=0} \gtrsim 4$ at $z \simeq 2$, with the inequality depending on the unknown stellar mass-to-light ratios of their galaxies. A similar result was obtained by McLure et al. (2006), combining $M_{\text {bulge }}$ of radio galaxies with $M_{\mathrm{bh}}$ of radio-loud QSOs, assuming these to be the same class of objects. An extremely large evolution in $\mu(z)$ 
was claimed by Shields et al. (2006) who used CO emission line widths as surrogates for stellar velocity dispersion and found that $\mu_{z=4} / \mu_{z=0} \sim 50$ at $z \simeq 4$. On the other hand, Lauer et al. (2007) recently demonstrated that comparing high-redshift quasar measurements with low-redshift galaxies can lead to systematically overestimating the amount of evolution in $\mu$. Our experimental setup is clearly affected by such biases, as the quasars were drawn from a survey for very luminous quasars. From Fig. 6 in Lauer et al. (2007) we estimate that our value of $\mu$ may be biased by up to 0.5 dex towards high values. Taking this bias into account then there is still a systematic offset of our $\mu$ values to the local one, but it is now well below our $1 \sigma$ errors.

\section{Conclusions}

We have presented rest-frame $B$ and $V$ imaging of QSO host galaxies, allowing us for the first time to measure an optical $B-V$ colour for at least some of our targets. We found these galaxies to show rather, but not extremely, blue colours, indicative of either an enormous burst of star formation a few hundred Myr ago, or of ongoing star formation on a much lower but still substantial level. Clearly, a single colour cannot differentiate between these options. It might be useful to search for $\mathrm{CO}$ emission in these QSOs which, if found, could be taken as evidence for ongoing star formation.

At least one of our QSOs is embedded in a highly asymmetric host galaxy structure, indicative of gravitational interaction or merging. This asymmetric structure is more prominent in the $H$ (rest-frame $B$ ) than in the $K_{\mathrm{s}}$ (rest-frame $V$ ) band image, which suggests that the blue light from young stars comes preferentially from this structure.

Our observations show that high-luminosity QSOs $\left(M_{B} \lesssim\right.$ $-28)$ at $z \sim 3$ already lie in very massive galaxies of several times $10^{11} M_{\odot}$. Such massive structures are rare at high $z$, but not exceedingly rare: following Fontana et al. (2006), the space density of galaxies with stellar masses above $3 \times 10^{11} M_{\odot}$ at $z \sim 3$ is of the order of $10^{-5}-10^{-6} \mathrm{Mpc}^{-3}$, still substantially higher than the number density of luminous QSOs (Wisotzki 2000). Thus, even at the peak of the cosmic QSO activity, only a small fraction of galaxies is growing their black holes at near Eddington rates.

By combining stellar bulge masses and virial black hole masses for two objects, we could estimate the ratio $\mu$ of these quantities in individual objects. We found a marginal excess of $\mu(z=3)$ compared to the local relation. Taking into account the substantial uncertainties, and considering recent analyses of systematic biases, however, our results are still consistent with no evolution in the black hole mass-bulge mass ratio. Still we believe that the approach as such is promising, and with larger samples and more accurate measurements, obtained at several redshifts, it has the potential to significantly constrain the degree of co-evolution between black holes and hosting bulges.

Acknowledgements. We thank Dr. Asmus Boehm, Dr. Isabelle Gavignaud and Dr. Chien Y. Peng for illuminating discussions. We are gratful to the anonymous referee who made numerous suggestions on how to improve this paper.

\section{References}

Abraham, R. G., Crawford, C. S., \& McHardy, I. M. 1992, ApJ, 401, 474 Bell, E. F., McIntosh, D. H., Katz, N., \& Weinberg, M. D. 2003, ApJS, 149, 289 Collin, S., Kawaguchi, T., Peterson, B. M., \& Vestergaard, M. 2006, A\&A, 456, 75

Dunlop, J. S., McLure, R. J., Kukula, M. J., et al. 2003, MNRAS, 340, 1095 Falomo, R., Kotilainen, J. K., Pagani, C., Scarpa, R., \& Treves, A. 2004, ApJ, 604,495

Falomo, R., Kotilainen, J. K., Scarpa, R., \& Treves, A. 2005, A\&A, 434, 469 Floyd, D. J. E., Kukula, M. J., Dunlop, J. S., et al. 2004, MNRAS, 355, 196 Fontana, A., Salimbeni, S., Grazian, A., et al. 2006, A\&A, 459, 745 Häring, N., \& Rix, H.-W. 2004, ApJ, 604, L89

Jahnke, K., Kuhlbrodt, B., \& Wisotzki, L. 2004a, MNRAS, 352, 399 Jahnke, K., Sánchez, S. F., Wisotzki, L., et al. 2004b, ApJ, 614, 568 Jorgensen, I., Franx, M., \& Kjaergaard, P. 1996, MNRAS, 280, 167 Kaspi, S., Smith, P. S., Netzer, H., et al. 2000, ApJ, 533, 631

Kauffmann, G., Heckman, T. M., Tremonti, C., et al. 2003, MNRAS, 346, 1055 Kollmeier, J. A., Onken, C. A., Kochanek, C. S., et al. 2006, ApJ, 648, 128 Kuhlbrodt, B., Wisotzki, L., \& Jahnke, K. 2004, MNRAS, 349, 1027

Kuhlbrodt, B., Örndahl, E., Wisotzki, L., \& Jahnke, K. 2005, AAP, 439, 497 Kukula, M. J., Dunlop, J. S., McLure, R. J., et al. 2001, MNRAS, 326, 1533 Lauer, T. R., Tremaine, S., Richstone, D., \& Faber, S. M. 2007 [arXiv: 0705.4103]

Magorrian, J., Tremaine, S., Richstone, D., et al. 1998, AJ, 115, 2285

McLeod, K. K., \& Rieke, G. H. 1995, ApJ, 441, 96

McLeod, K. K., Rieke, G. H., \& Storrie-Lombardi, L. J. 1999, ApJ, 511, L67

McLure, R. J., \& Jarvis, M. J. 2002, MNRAS, 337, 109

McLure, R. J., Kukula, M. J., Dunlop, J. S., et al. 1999, MNRAS, 308, 377

McLure, R. J., Jarvis, M. J., Targett, T. A., Dunlop, J. S., \& Best, P. N. 2006, MNRAS, 368, 1395

Peng, C. Y., Impey, C. D., Ho, L. C., Barton, E. J., \& Rix, H.-W. 2006a, ApJ, 640, 114

Peng, C. Y., Impey, C. D., Rix, H.-W., et al. 2006b, ApJ, 649, 616

Persson, S. E., Murphy, D. C., Krzeminski, W., Roth, M., \& Rieke, M. J. 1998, AJ, 116, 2475

Peterson, B. M., \& Wandel, A. 2000, ApJ, 540, L13

Ridgway, S. E., Heckman, T. M., Calzetti, D., \& Lehnert, M. 2001, ApJ, 550, 122

Sánchez, S. F., Jahnke, K., Wisotzki, L., et al. 2004, ApJ, 614, 586

Shields, G. A., Menezes, K. L., Massart, C. A., \& Vanden Bout, P. 2006, ApJ, 641,683

Vestergaard, M. 2002, ApJ, 571, 733

Vestergaard, M. 2004, in ASP Conf. Ser., 311, AGN Physics with the Sloan Digital Sky Survey, 69

Vestergaard, M., \& Peterson, B. M. 2006, ApJ, 641, 689

Wisotzki, L. 2000, A\&A, 353, 853

Wisotzki, L., Christlieb, N., Bade, N., et al. 2000, AAP, 358, 77 